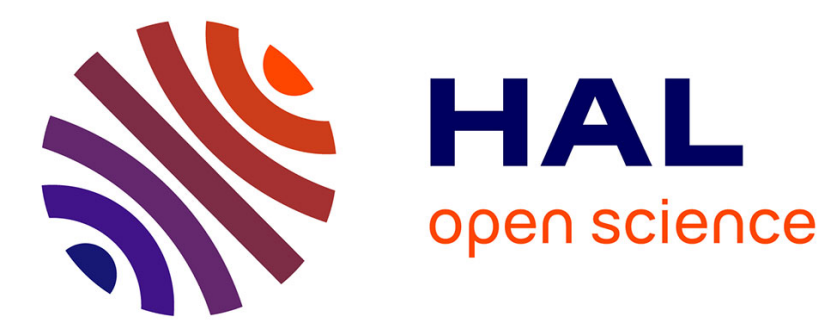

\title{
Spatial Modulation for Multiple-Antenna Communication
}

Marco Di Renzo, Harald Haas, Ali Ghrayeb, Lajos Hanzo, Shinya Sugiura

\section{To cite this version:}

Marco Di Renzo, Harald Haas, Ali Ghrayeb, Lajos Hanzo, Shinya Sugiura. Spatial Modulation for Multiple-Antenna Communication. John G. Webster. Wiley Encyclopedia of Electrical and Electronics Engineering, 2016, 10.1002/047134608X.W8327 . hal-02324436

\section{HAL Id: hal-02324436 \\ https://hal-centralesupelec.archives-ouvertes.fr/hal-02324436}

Submitted on 21 Oct 2019

HAL is a multi-disciplinary open access archive for the deposit and dissemination of scientific research documents, whether they are published or not. The documents may come from teaching and research institutions in France or abroad, or from public or private research centers.
L'archive ouverte pluridisciplinaire HAL, est destinée au dépôt et à la diffusion de documents scientifiques de niveau recherche, publiés ou non, émanant des établissements d'enseignement et de recherche français ou étrangers, des laboratoires publics ou privés. 
See discussions, stats, and author profiles for this publication at: https://www.researchgate.net/publication/310599151

\section{Spatial Modulation for Multiple-Antenna Communication}

Chapter $\cdot$ November 2016

DOI: 10.1002/047134608X.w8327

\section{5 authors, including:}

$$
\text { Marco Di Renzo }
$$

French National Centre for Scientific Research

278 PUBLICATIONS 6,456 CITATIONS

SEE PROFILE

L. Hanzo

University of Southampton

2,411 PUBLICATIONS 33,523 CITATIONS

SEE PROFILE

Some of the authors of this publication are also working on these related projects:

spatial modulation View project

Project Ultra-reliable and low-latency communication (URLLC) systems View project 


\title{
Spatial Modulation for Multiple-Antenna Communication
}

\author{
Marco Di Renzo, Senior Member, IEEE, Harald Haas, Member, IEEE, \\ Ali Ghrayeb, Senior Member, IEEE, Shinya Sugiura, Senior Member, IEEE, and \\ Lajos Hanzo, Fellow, IEEE
}

\begin{abstract}
The key challenge of future mobile communication networks is to strike an attractive compromise between the wireless network's area spectral efficiency and energy efficiency. This necessitates new approaches to wireless system design, embracing a rich body of existing knowledge - especially in the field of Multiple-Input-Multiple-Output (MIMO) techniques. Hence in this chapter, we describe Spatial Modulation (SM), which constitutes a promising new concept of wireless system design conceived for reducing the number of high-cost RF front-ends compared to other MIMO implementations. The concept of SM has recently established itself as a beneficial transmission paradigm, subsuming numerous members of the MIMO system-family. Research activities on SM have reached sufficient maturity to motivate its comparison against state-of-the-art MIMO systems, as well as to inspire its application in emerging wireless communication systems. Furthermore, it has received sufficient research attention to motivate its implemention in testbeds and it holds the promise of stimulating further vigorous interdisciplinary research in the years to come, especially in the emerging market of the Internet of Things (IoT).
\end{abstract}

\section{Index Terms}

Manuscript December 22, 2015.

M. Di Renzo is with the Laboratoire des Signaux et Systèmes, CNRS, CentraleSupélec, Univ Paris Sud, Université ParisSaclay, 3 rue Joliot Curie, Plateau du Moulon, 91192, Gif-sur-Yvette, France (e-mail: marco.direnzo@12s.centralesupelec.fr).

H. Haas is with The University of Edinburgh, Institute for Digital Communications (IDCOM), Mayfield Road, Edinburgh, EH9 3JL, United Kingdom (e-mail: h.haas@ed.ac.uk).

A. Ghrayeb is with the Department of Electrical and Computer Engineering, Texas A\&M University at Qatar (e-mail: ali.ghrayeb@qatar.tamu.edu).

S. Sugiura is with the Department of Computer and Information Sciences, Tokyo University of Agriculture and Technology, Tokyo, 184-8588, Japan (e-mail: sugiura@ieee.org).

L. Hanzo is with The University of Southampton, Communications, Signal Processing and Control Group, Department of Electronics and Computer Science, SO17 1BJ, United Kingdom (e-mail: lh@ecs.soton.ac.uk). 
Multiple-antenna systems, spatial modulation, few-RF multiple-antenna design, cellular networks, connected objects.

\section{INTRODUCTION}

It is widely recognized that the Long Term Evolution (LTE) is the de facto physical-layer standard of fourth generation $(4 \mathrm{G})$ cellular networks [1], [2]. The power consumption of the Information and Communication Technology (ICT) sector in the next decade will highly depend on the Energy Efficiency (EE) of this physical-layer standard [3], [4]. At the current stage, however, the LTE standard may be deemed to be conceived, designed and optimized based on the Spectral Efficiency (SE), with limited consideration of the associated EE issues [5]. Hence the primary focus has been on achieving high data rates, without giving much cognizance to EE and to the implementation complexity, especially at the physical-layer. This approach, unfortunately, is no longer acceptable for future services relying on cellular networks. The recent research efforts and the decision to standardize a new narrow-band radio technology for the Internet of Things (IoT) confirm this paradigm change. The new radio access network standard is known as the Narrow-Band IoT (NB-IoT) recommendation, which is expected to provide improved indoor coverage, to support a massive number of low-throughput devices, to guarantee a limited delay, to facilitate a low device cost as well as power consumption and so on [6]. This technology is expected to operate "in-band", relying on the resource blocks of a standard LTE carrier, or in the unused resource blocks within a LTE carrier's guard-band, or in a "stand-alone" mode for emloyment in dedicated spectrum.

The operational LTE physical-layer standard relies on Multiple-Input-Multiple-Output (MIMO) techniques for enhancing the SE and for reducing the transmit power [7], [8]. MIMO aided techniques will undoubtedly find their way into the fifth generation (5G) of mobile services and applications. In emerging IoT applications, on the other hand, the so-called connected objects are expected to require modest data rates, a low power consumption, and a smaller form factor compared to those of typical mobile multi-media services. By contrast, they are expected to support higher data rates at a higher power consumption, and at a more bulky form factor compared to typical wireless sensor networks. Hence, new air-interface techniques have to be developed, which are capable of satisfying these new emerging requirements. Clearly, MIMO transmission and reception schemes will continue to play a vital role in these emerging 
application scenarios, in light of their inherent design flexibility. However, new MIMO paradigms are necessary.

In simple terms, the capacity of MIMO systems is proportional to $\min \left\{N_{t}, N_{r}\right\}$, where $N_{t}$ and $N_{r}$ represent the number of transmit and receive antennas. This implies that the throughput may be increased linearly with the number of antennas. As a consequence, MIMO techniques can provide high data rates without any bandwidth-expansion and without increasing the transmit power [7]. However, in practice, MIMO systems rely on a multiplicity of associated circuits, such as power amplifiers, RF front-ends, mixers, synthesizers, filters, etc., which substantially increase the circuit power dissipation of all network elements. More explicitly, recent studies have clearly shown that the EE gain of MIMO transmission increases with the number of antennas, provided that only the transmit power is taken into account and their circuit power dissipation is neglected [9], [10], [11]. On the other hand, the EE gain of MIMO transmission remains modest and decreases with the number of active transmit antennas, if realistic power consumption models are considered [12]. These results highlight that the design of energy efficient and lowcomplexity MIMO transmission is an open research problem at the time of writing, especially in the context of IoT applications. In fact, many system parameters have to be considered, such as the bandwidth, the transmit power, the number of active transmit/receive antennas, the number of active devices, etc., which all contribute to the fundamental transmit power vs. circuit power dissipation and multiplexing gain vs. inter-user interference trade-offs. As a result, while the SE advantages of MIMO transmission are widely recognized, its EE potential is not well understood.

Conventional MIMO transmission takes advantage of all the antennas available at the transmitter, by simultaneously transmitting multiple data streams from all of them. Thus, all transmit antennas are activated at any time instance. By appropriately choosing the transmission/precoding matrices, multiplexing (higher data rates) and/or transmit-diversity (better link reliability) gains can be obtained using MIMO systems. The reason behind this choice is that simultaneously activating all transmit antennas results in an improved SE [8]. However, unfortunately, this choice does not lead to optimizing the total EE [12]. In fact, multiple RF chains are required at both the transmitter and receiver for simultaneously transmitting many data streams, each of which requires an independent power amplifier that is known to dissipate the majority of the power consumed [3]. These considerations imply that a major challenge of next-generation MIMO-aided cellular networks is the design of multi-antenna transmission schemes with a limited 
number of RF front-ends, aiming for reducing the complexity, for relaxing both the inter-antenna synchronization requirements, the inter-channel interference, as well as the signal processing complexity and the form factor of the network elements, whilst aiming for improving the SE compared to single-antenna transmission and for reducing the total power consumption compared to conventional multiple-antenna aided transmission.

In this context, the design of MIMO transmission schemes that exploit fewer RF chains compared to the number of available radiating elements is currently emerging as a promising research field [13]. Usually, this family of MIMO designs is referred to as a single-RF MIMO. Suffice to say, however that there are also various compromise-schemes, which rely on diverse antennaselection schemes, where a specific fraction of the antennas is activated, which determines the number of RF-chains necessitated. Hence in parlance we can refer to these schemes as single-RF, full-RF and fractional-RF arrangements.

To elaborate a little further, the fundamental idea behind the fractional-RF aided MIMO schemes is to attain spatial multiplexing and/or transmit-diversity gains with the aid of many antenna-elements, where only a fraction of the antenna-elements - or possibly just a single antenna-element - is activated at the transmitter at any modulation instant. The rationale behind the full-RF to fractional-RF paradigm shift in MIMO design originates from the consideration that multiple transmit antennas (radiating elements) may be accommodated at both the transmitters and receivers, bearing in mind that the complexity and power consumption/dissipation of MIMO transmission is mainly determined by the number of simultaneously active transmit antennas, i.e., by the number of RF chains [9], [10].

Fueled by these considerations, SM has recently established itself as a promising transmission concept, which belongs to the fractional-RF MIMO wireless system family, whilst exploiting the availability of multiple antennas in a novel fashion compared to state-of-the-art high-complexity and power-hungry classic MIMO systems [14]. In simple terms, SM can be regarded as a MIMO concept that possesses a larger set of radiating elements than the number of transmit-electronics chains. SM-MIMO takes advantage of the entire antenna-array at the transmitter, whilst using a limited number of RF chains. The main distinguishing feature of SM-MIMO is that it maps additional information bits onto a "SM constellation diagram", where each constellation element is constituted by either a single one or a subset of antenna-elements. These unique characteristics result in high-rate MIMO implementations relying on a reduced signal processing and circuit 
complexity, as well as an improved EE. Recent analytical and simulation studies have shown that SM-MIMOs have the inherent potential of outperforming many state-of-the-art MIMO schemes under the fair assumption that the number of RF chains is the same.

In a nutshell, the rationale behind SM-MIMO communications designed for spectral- and energy-efficient cellular networks is supported by a pair of main pillars: 1) Given the performance constraints, they minimize the number of activated antenna-elements in order to increase the EE by reducing the circuit power consumption and the form factor of the devices; 2) Given the implementation and size constraints, they maximize the number of radiating antenna-elements in order to increase both the SE and the EE by reducing the transmit power consumption. This is realized by capitalizing on the multiplexing gain introduced by mapping additional bits onto the so-called "SM constellation diagram" constituted by the antennas.

Against this back-drop, in this chapter the concept of SM-MIMO is introduced and its achievable performance in terms of its error probability, signal processing complexity and energy efficiency is discussed. Finally, experimental results relying on a testbed are illustrated in order to substantiate the feasibility of this emerging concept.

\section{A Historical Perspective}

Although SM-MIMO has received widespread attention from the research community only in the last few years (about 11 papers were available in the open technical literature in 2008), it is a 14-year old technology. In this section, we briefly describe the history of SM-MIMO research with a focus on some pioneering papers, where the concept of SM is first proposed and studied. From this short glimpse into the historical development of SM-MIMO, it is evident that during the years 2001-2008 various researchers independently developed transmission concepts closely-related to the SM-MIMO scheme, which were nicknamed with different acronyms.

The "space modulation" principle appeared for the first time in 2001 [15]. This scheme was termed as Space Shift Keying (SSK) modulation and it exploits the differences in the signals received from different Transmit Antennas (TAs) to discriminate the transmitted information messages. In [15], a twin-antenna MIMO setup providing 1 bit-per-channel-use (bpcu) transmission was investigated. The information bits are encoded by keeping one TA active all the time, while activating the second TA only for one of the two possible information bits. The authors also proposed a twin-antenna MIMO setup that combines spatial and binary Phase Shift Keying 
(PSK) modulation, thus providing 2 bpcu transmission. The scheme employs 2-PSK modulation, when the TAs are active.

A year later in 2002, Haas et al. [16] the authors proposed a multi-antenna modulation scheme, where a number of bits that is equal to that of the TA-elements is multiplexed in an orthogonal fashion. A special property of the encoding scheme is that only one out of the available TAs is active in every channel use. The scheme is developed for 2-PSK modulation and some bits are used as parity checks. The authors show that it provides the same spectral efficiency as 8-PSK, but with a better error probability.

Two years later in 2004, Song et al. [17] proposed a modulation scheme termed as the "channel hopping technique," which is exactly what is known today as SM-MIMO. The idea is portrayed in [17, Fig. 1], and it foresees the transmission of two information streams: the first is explicitly transmitted by using conventional PSK or Quadrature Amplitude Modulation (QAM) and the second is implicitly transmitted by activating a single TA of the available antenna-array.

In 2005, Mesleh et al. [18] independently proposed the same modulation scheme as in [17]. The main motivation behind [18] is to develop a multi-antenna modulation scheme that avoids Inter-Antenna Interference (IAI), which is realized by activating a single TA in every channel use and by implicitly conveying additional information bits using the TA switching process. The authors also show that the proposed scheme significantly relaxes the signal processing complexity at the receiver. In 2006, the same authors further investigated the scheme proposed in [18] and they used for the first time the terminology of "Spatial Modulation" to identify this transmission mechanism [19], [20], [21], [22].

Two years later in 2008, various papers were published by improving and further investigating the SM-MIMO concepts presented in the previous years. Yang and Jiao et al. [23] study the channel capacity, where the parlance of Information Guided Channel Hopping (IGCH) is coined. It is shown that IGCH provides a better spectral efficiencies than Orthogonal Space Time Block Codes (OSTBCs). In [24], the SM-MIMO concept introduced in [18], [19], [20], [21], [22] is comprehensively studied by using a low-complexity two-step demodulator. In [25], the authors develop the Maximum Likelihood (ML) optimum demodulator for SM-MIMO and they show that some performance improvements can be expected compared to the sub-optimal demodulator introduced in [24]. In [26], the SM-MIMO scheme is simplified by generalizing the SSK concept originally proposed in [15] to arbitrary numbers of TAs. In particular, only the spatial- 
constellation diagram is used to transmit information bits. This encoding scheme is today known as SSK-MIMO. In [27], a framework is introduced for the performance analysis of SM-MIMO by using results from ordered statistics. In [28], the authors extend [26] by allowing more than one TA to be active in every channel use and by encoding the information bits onto various combinations of multiple active TAs. They show that for the same number of TA-elements the rate can be improved at the cost of increasing the number of RF chains, whilst tolerating some performance loss. This modulation scheme is referred to as Generalized SSK (GSSK).

More recently, in [29], [30], [31], [32], [33] the issue of fast RF switching has been addressed in the context of SM/SSK, which is a major practical implementation challenge of these techniques. Due to the specific encoding mechanism of SM/SSK, the active transmit antenna is switched at each new channel use, which requires an RF switch operating at the symbol rate. While fast RF switches with low insertion loss and with good isolation properties are available in the literature for a wide range of frequency bands, high-speed RF switches still constitute a critical part of the transmitter design [34]. To this end, single-RF MIMO systems have been implemented using the concept of reconfigurable antennas (RAs). Indeed, the implementation of SM/SSK using a single RA with multiple transmission states allows for replacing the switching amongst RF chains by a switching operation amongst antenna states. The latter process is associated with modifying the surface current distribution within the RA and thus it is associated with a lower complexity and lower cost than switching amongst RF chains during each transmission period.

At the time of writing, research on SM-MIMO is well established and hundreds of research contributions are available in the open technical literature. The interested readers are invited to consult recent survey papers in this field of research [14], [34], [35], [36], [37].

\section{OPERATING PRINCIPLE}

\section{A. How It Works}

In this section, we introduce the SM-MIMO concept illustrating it with the aid of some simple examples. We denote by $N_{t}$ and $N_{r}$ the number TAs and Receive Antennas (RAs), respectively. The cardinality of the signal-constellation diagram is denoted by $M$. Either PSK or QAM are considered. In general, $N_{t}, N_{r}$ and $M$ can be chosen independently of each other. At the receiver, ML-optimum demodulation is considered. Thus, $N_{r}$ can be chosen independently of $N_{t}$ [7]. For ease of presentation, we assume $N_{t}=2^{n_{t}}$ and $M=2^{m}$ with $n_{t}$ and $m$ being 

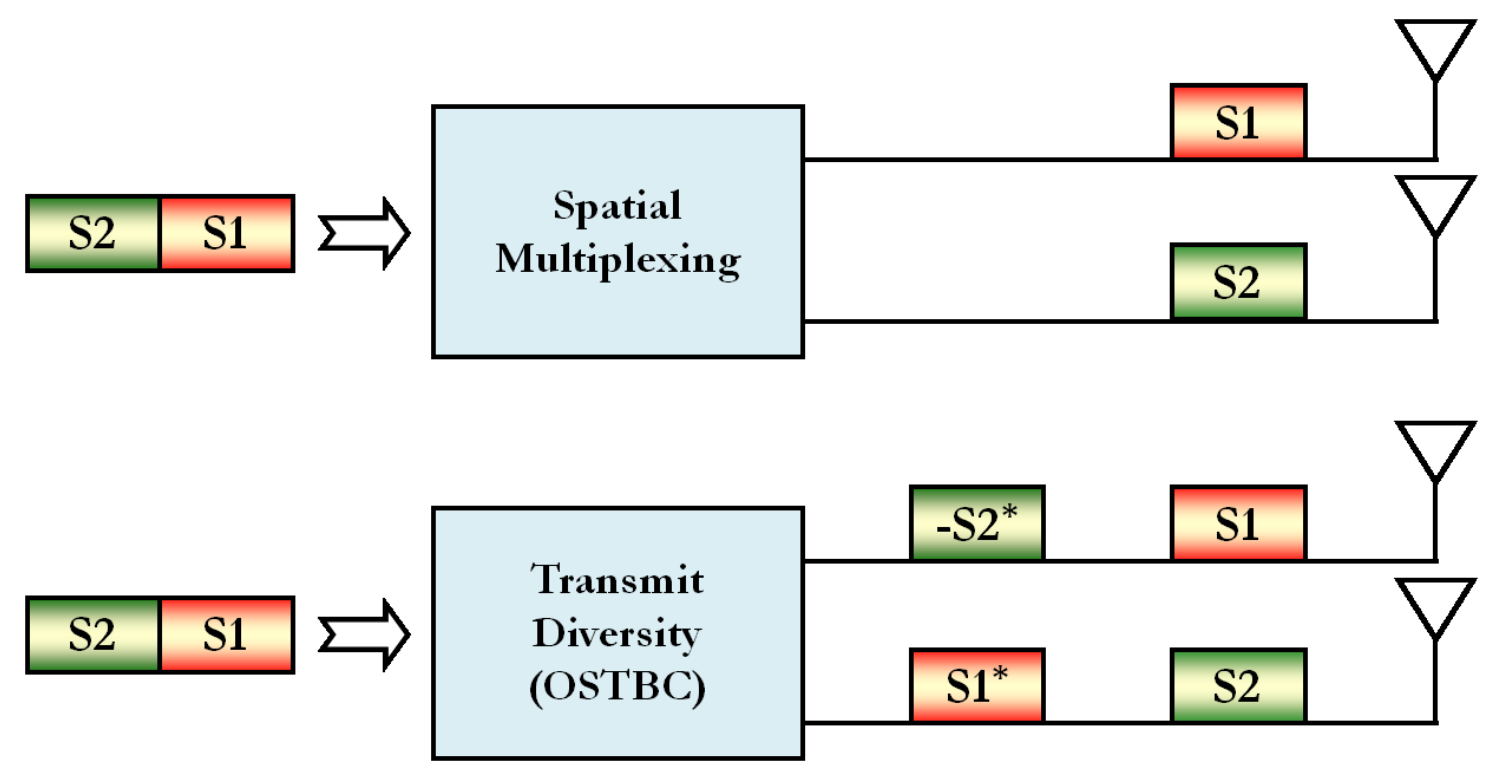

(b)

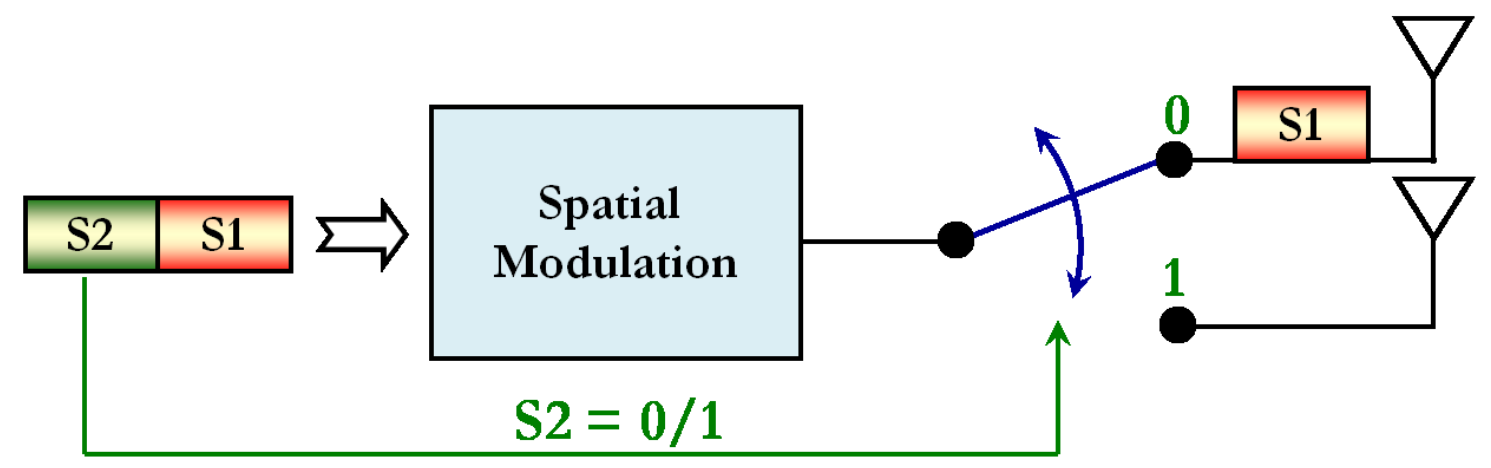

(c)

Fig. 1. Illustration of three MIMO concepts: (a) spatial-multiplexing; (b) transmit-diversity; and (c) spatial modulation. Reproduced with permission from [34].

two positive integers. For ease of illustration, a single RF front-end is supposed to be available at the transmitter (single-RF MIMO).

In Fig. 1, the SM-MIMO concept is illustrated for $N_{t}=M=2$ and it is compared to the conventional Spatial Multiplexing (SMX) scheme [7, Sec. II] and to the OSTBC scheme designed for transmit-diversity [7, Sec. III]. In the latter case, the Alamouti scheme is considered as an example [38].

1) In SMX-MIMO, two PSK/QAM symbols $\left(S_{1}\right.$ and $\left.S_{2}\right)$ are simultaneously transmitted from a pair of TAs in a single channel use. For arbitrary $N_{t}$ and $M$, the rate of SMX is $R_{\mathrm{SMX}}=N_{t} \log _{2}(M)$ bpcu [7, Sec. II].

2) In OSTBC-MIMO, two PSK/QAM symbols $\left(S_{1}\right.$ and $\left.S_{2}\right)$ are first encoded and then simul- 
taneously transmitted from a pair of TAs in two channel uses. For arbitrary $N_{t}$ and $M$, the rate of OSTBC is $R_{\mathrm{OSTBC}}=R_{c} \log _{2}(M)$ bpcu, where $R_{c}=N_{\mathrm{M}} / N_{c u} \leq 1$ is the rate of the space-time block code and $N_{\mathrm{M}}$ is the number of information symbols transmitted in $N_{c u}$ channel uses [7, Sec. III], [39]. If, as shown in Fig. 1, the Alamouti code is chosen, then we have $R_{c}=1$ [38].

3) In SM-MIMO, only one $\left(S_{1}\right)$ out of the two symbols is explicitly transmitted, while the other symbol $\left(S_{2}\right)$ is implicitly transmitted by determining the index of the active TA in each channel use. In other words, in SM-MIMO the information symbols are modulated onto two information carrying units: 1) a PSK/QAM symbol; and 2) a single active TA via an information-driven antenna-switching mechanism. For arbitrary $N_{t}$ and $M$, the rate of $\mathrm{SM}$ is $R_{\mathrm{SM}}=\log _{2}(M)+\log _{2}\left(N_{t}\right)$ bpcu [14], [24].

In Fig. 2 and Fig. 3, the encoding principle of SM-MIMO is illustrated for $N_{t}=M=4$ by considering two generic channel uses, where the concept of "SM or spatial-constellation diagram" is also introduced. The rate of this MIMO setup is $R_{\mathrm{SM}}=\log _{2}(M)+\log _{2}\left(N_{t}\right)=4$ bpcu, hence the encoder processes the information bits in blocks of 4 bits each. In the first channel use shown in Fig. 2, the block of bits to be encoded is " 1100 ". The first $\log _{2}\left(N_{t}\right)=2$ bits, "11," determine the single active TA $\left(\mathrm{TX}_{3}\right)$, while the second $\log _{2}(M)=2$ bits, " 00 ," determine the transmitted PSK/QAM symbol. Likewise, in the second channel use shown in Fig. 3, the block of bits to be encoded is " 0001 ". The first $\log _{2}\left(N_{t}\right)=2$ bits, "00," determine the single active TA $\left(\mathrm{TX}_{0}\right)$, while the second $\log _{2}(M)=2$ bits, " 01 ," determine the transmitted PSK/QAM symbol.

The illustrations shown in Fig. 2 and Fig. 3 highlight a pair of unique characteristics of SM-MIMO:

1) The activated TA may change every channel use according to the input information bits. Thus, TA switching is an effective way of mapping the information bits to TA indices and of increasing the transmission rate.

2) The information bits are modulated onto a three-dimensional constellation diagram, which generalizes the known two-dimensional (complex-valued) signal-constellation diagram of PSK/QAM modulation schemes. The third dimension is provided by the antenna-array, where some of the bits are mapped to the TAs. Again, in SM-MIMO research, this third 


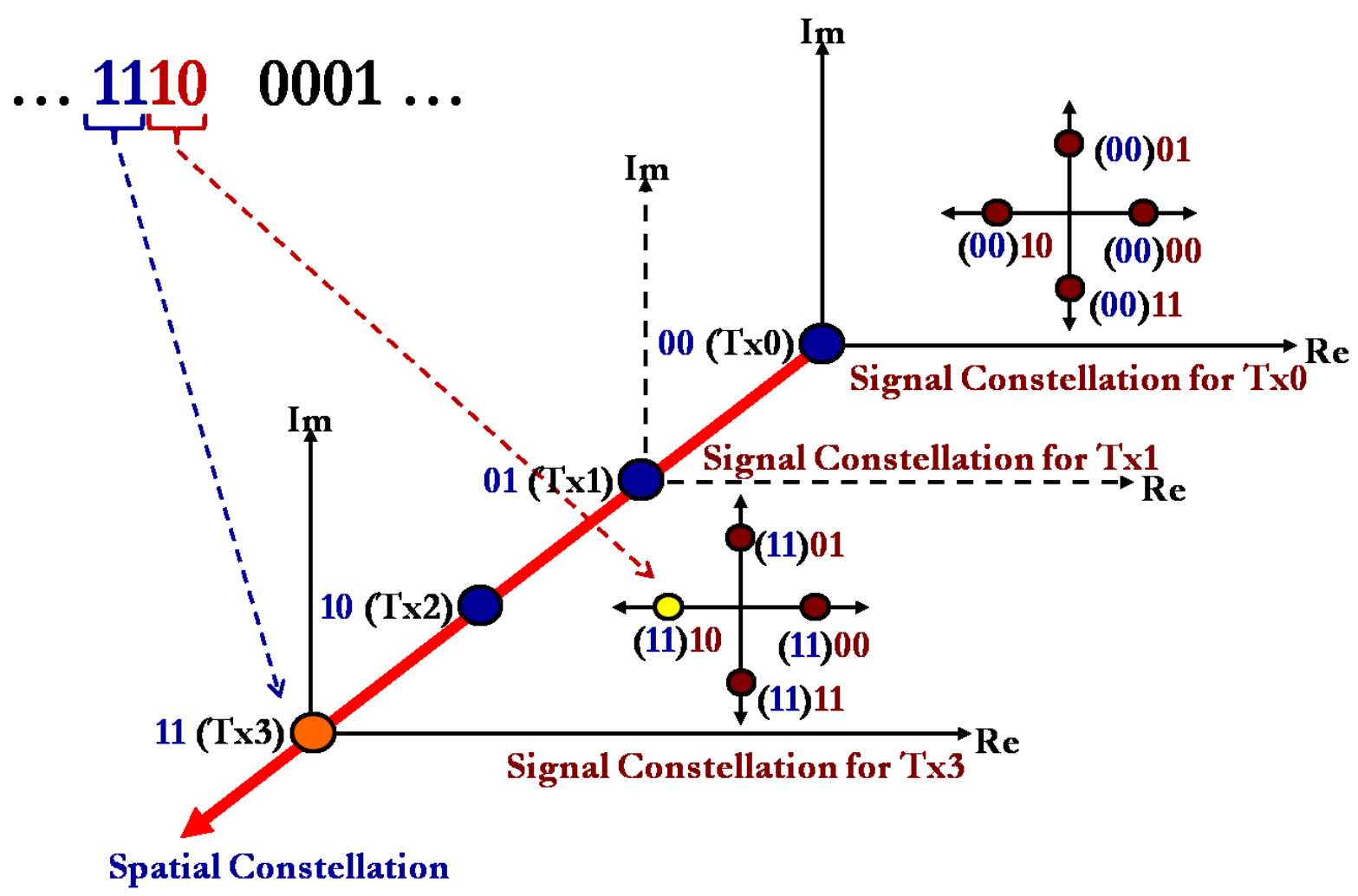

Fig. 2. Illustration of the three-dimensional encoding of spatial modulation (first channel use) Reproduced from [34] with permission.

dimension is often termed as the "spatial-constellation diagram" [14].

In simple mathematical terms, the signal model of SM-MIMO, assuming a frequency-flat channel model, is as follows:

$$
\mathbf{y}=\mathbf{H x}+\mathbf{n}
$$

where: i) $\mathbf{y} \in \mathbb{C}^{N_{r} \times 1}$ is the complex received vector; ii) $\mathbf{H} \in \mathbb{C}^{N_{r} \times N_{t}}$ is the complex channel matrix; iii) $\mathbf{n} \in \mathbb{C}^{N_{r} \times 1}$ is the complex Additive White Gaussian Noise (AWGN) at the receiver; and iv) $\mathbf{x}=\mathbf{e} s \in \mathbb{C}^{N_{t} \times 1}$ is the complex modulated vector with $s \in \mathcal{M} \subseteq \mathbb{C}^{1 \times 1}$ being the complex (scalar) PSK/QAM modulated symbol belonging to the signal-constellation diagram and $\mathbf{e} \in \mathcal{A}$ being the $N_{t} \times 1$ vector belonging to the spatial-constellation diagram $\mathcal{A}$ as follows:

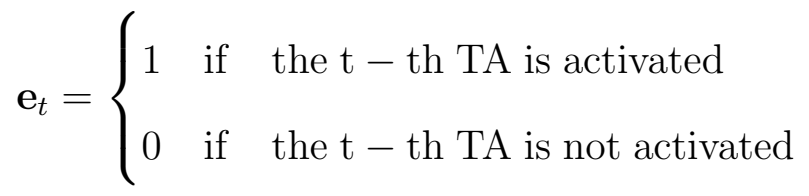




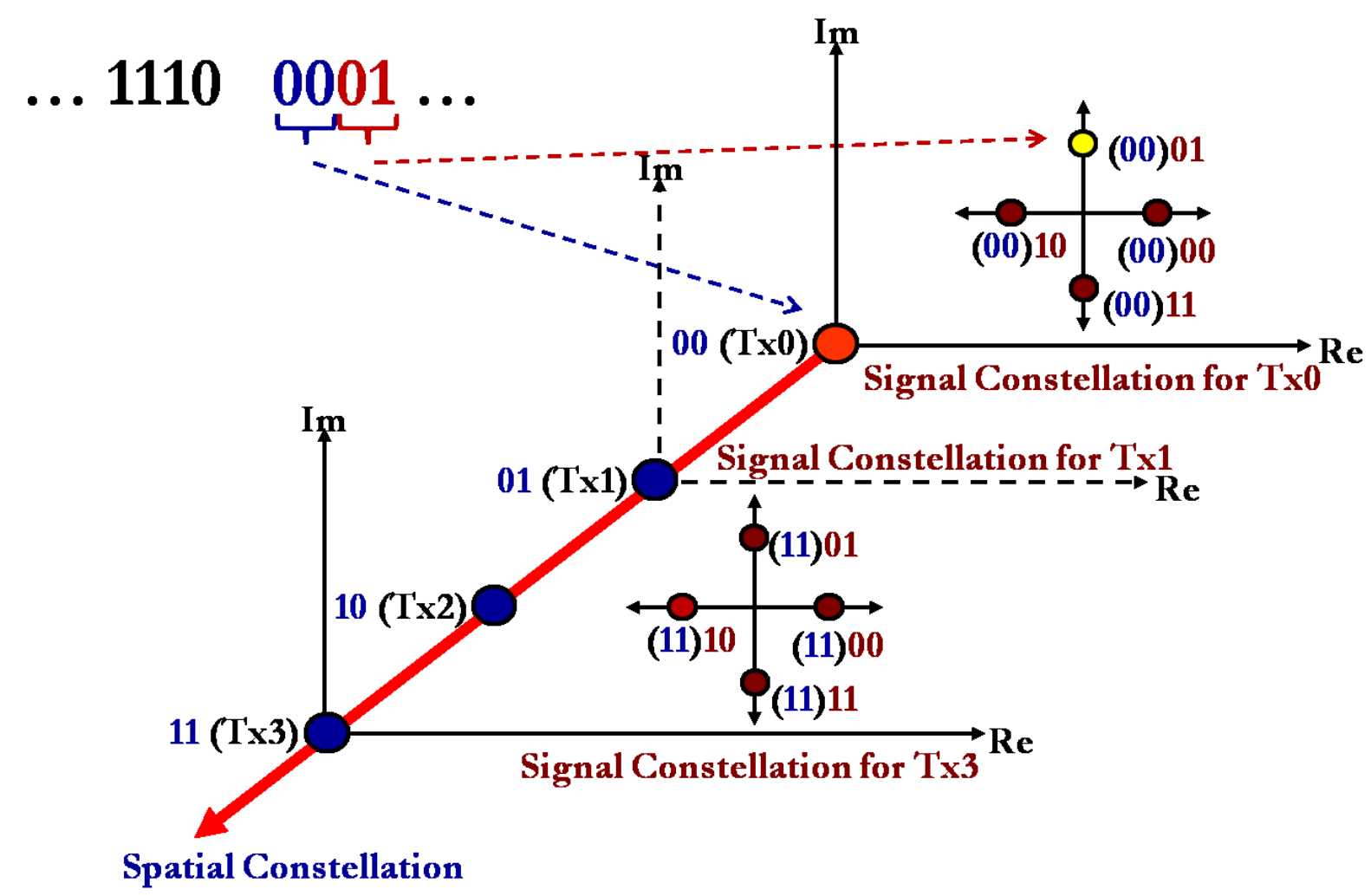

Fig. 3. Illustration of the three-dimensional encoding of spatial modulation (second channel use) Reproduced from [34] with permission.

where $\mathbf{e}_{t}$ is the $t$ th entry of $\mathbf{e}$ for $t=1,2, \ldots, N_{t}$. In other words, the points ( $N_{t}$-dimensional vectors) of the spatial-constellation diagram are the $N_{t}$ unit vectors of the natural basis of the $N_{t}$-dimensional Euclidean space.

If $N_{t}=1$, SM-MIMO reduces to conventional single-antenna communications, where the information bits are encoded only onto the signal-constellation diagram. In this case, the rate is $R_{0}=\log _{2}(M)$. On the other hand, if $M=1$ the information is encoded only onto the spatial-constellation diagram by providing a rate equal to $R_{\mathrm{SSK}}=\log _{2}\left(N_{t}\right)$. In the literature, this transmission scheme is known as SSK modulation [26]. In particular, SSK modulation is a MIMO scheme, where data transmission takes place only through the information-driven TA switching mechanism. It is apparent that SM-MIMO can be viewed as the combination of single-antenna PSK/QAM and SSK-MIMO modulations. 


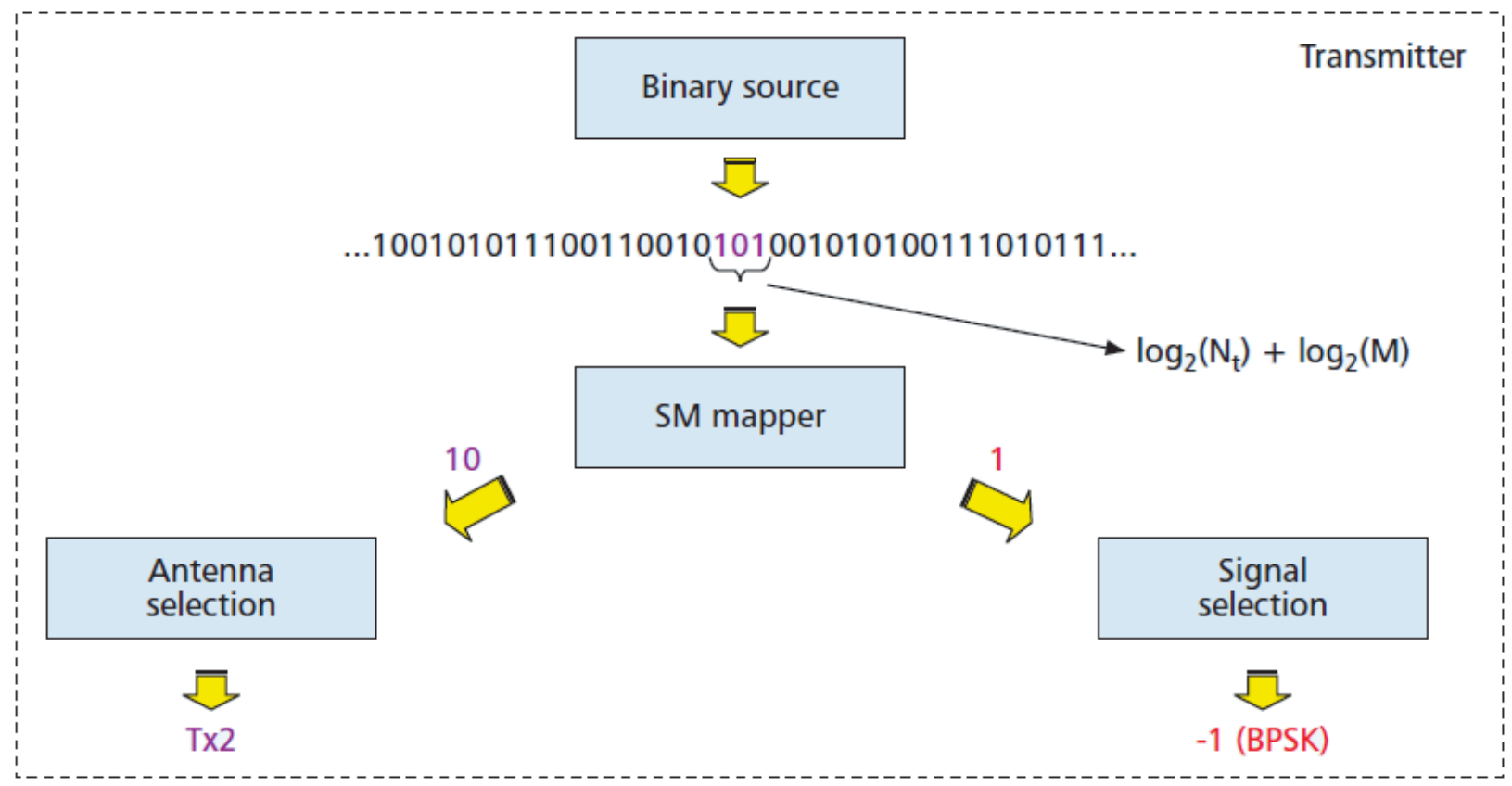

Fig. 4. Why it works (encoding). Reproduced from [14] with permission.

\section{B. Why It Works}

From Section III-A, we have learned that the fundamental operational principle of SM-MIMO relies on transmitting part of the information bits via an implicit information-driven antennaswitching mechanism. In this section, we provide some insights on how the receiver becomes capable of retrieving the information bits encoded into the TA-indices. This, in fact, showcases the underlying "essence" that equips SM-MIMO with its innate properties. Let us consider Figs. 4-6, which illustrates the bit-to-symbol mapping and the transmission process through the communication channel as well as the decoding process of SM-MIMO transmission, respectively.

Figure 4 is similar to Figs. 2 and 3, but $N_{t}=4$ and $M=2$ are considered for ease of illustration. Let "101" be the triplet of bits at the input of the SM modulator. Then, the TAelement $\mathrm{TX}_{2}$ is activated and the "-1" PSK bit is transmitted from it.

The signal emitted by the active TA then traverses through a generic wireless channel, whose channel impulse responses are illustrated in Fig. 5. Owing to the different spatial locations occupied by the TAs in the antenna-array, the signal transmitted by the active TA experiences different propagation conditions due to the different dispersive and reflecting environmental objects along any transmitter-to-receiver wireless links. As such, the same "-1" PSK symbol 


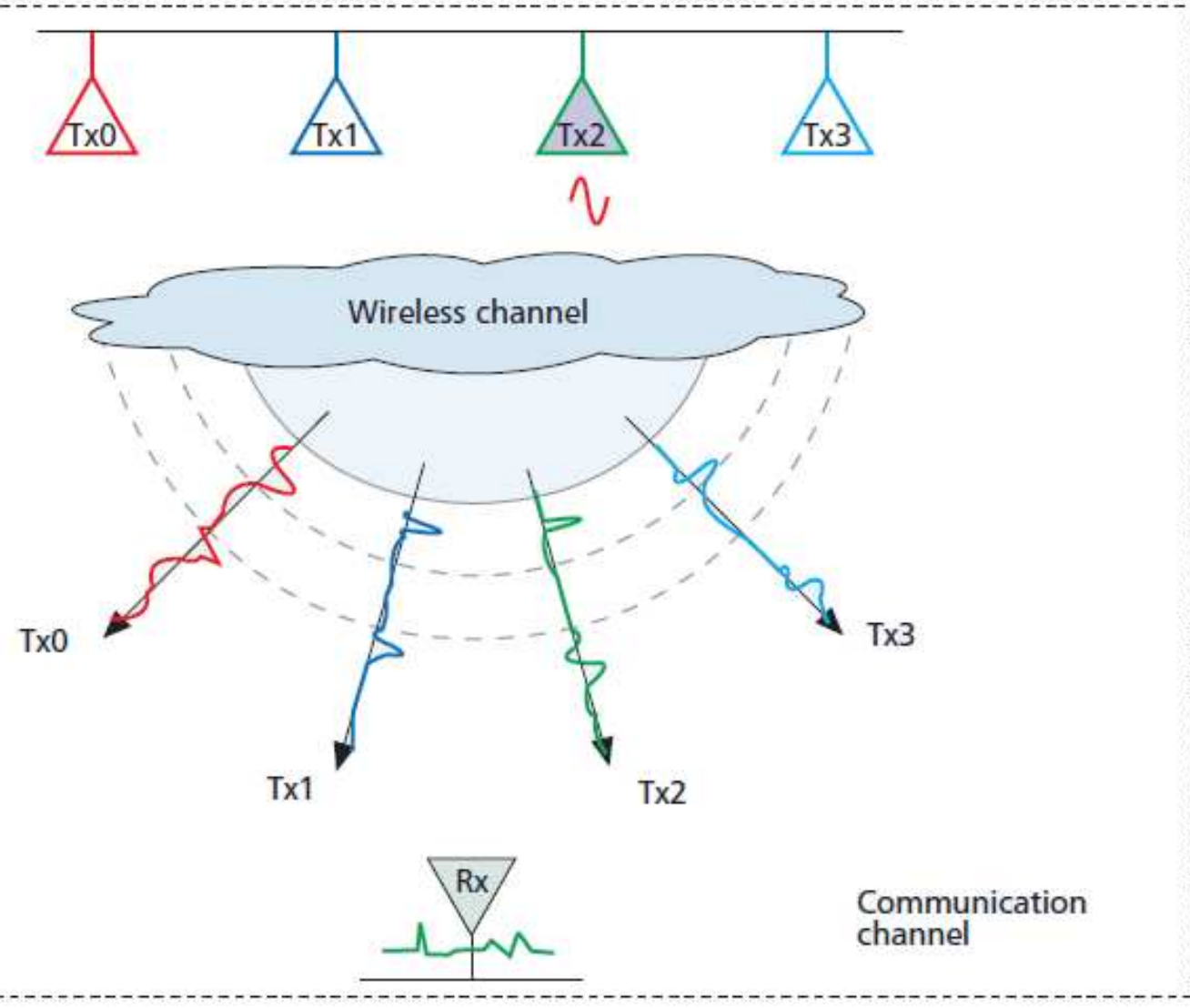

Fig. 5. Why it works (communication channel). Reproduced from [14] with permission.

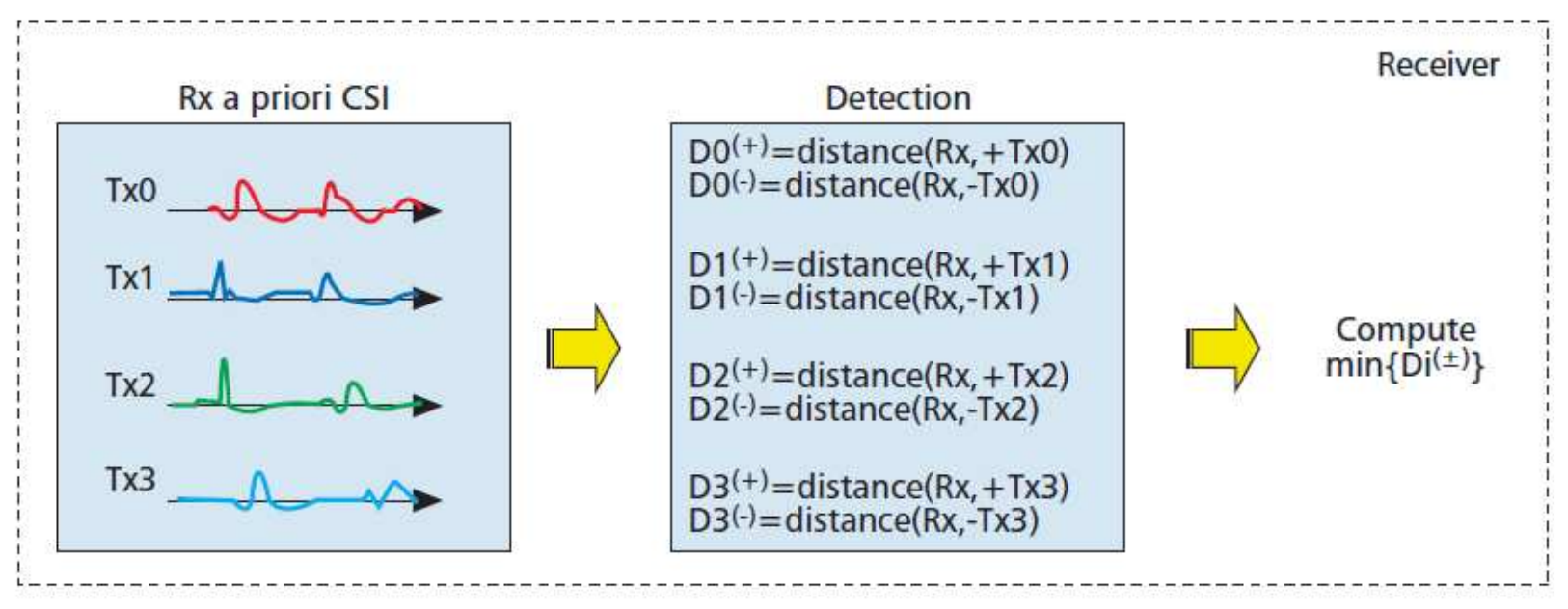

Fig. 6. Why it works (decoding). Reproduced from [14] with permission.

emitted by the TA-element $\mathrm{TX}_{2}$ travels through a communication channel, which introduces a 
specific "channel signature or fingerprint," i.e., the channel impulse response, that makes it unique compared to the same symbol emitted by any other TAs. This constitutes the fundamental essence of SM-MIMO: the more different the channel signatures/fingerprints are from each other, the simpler it becomes to distinguish the signals at the receiver. In simple terms, the communication channel may be deemed to play, especially for SSK-MIMO, the role of a "modulation unit," where the channel impulse responses are the actual messages being transmitted.

At the receiver, the demodulation unit exploits the unique fingerprint introduced by the wireless channel for retrieving the information bits. This is illustrated in Fig. 6, where a coherent demodulation scheme based on the minimum Euclidean distance is considered [25]. The receiver is assumed to be aware of the $N_{t}$ channel impulse responses, however the actual channel impulse response that is received in each channel use depends on the index of the active TA. The demodulator performs an exhaustive search among all the possible combinations of channel impulse responses and modulation symbols, and makes a decision in favor of the hypothesis associated with the lowest Euclidean distance. In a nutshell, due to the information-driven antenna-switching mechanism of SM-MIMO transmission, the $N_{t}$ channel impulse responses become part of the search space of the hypothesis-testing problem solved by the receiver. Based on the estimated channel impulse response, the demodulator is capable of retrieving the information bits associated with it. In summary, the essence of SM-MIMO transmission is all about exploiting the TA-specific property of the wireless channel, i.e., the uniqueness of each transmit-to-receive wireless link, for data communication.

\section{Achievable Performance}

In this section, we illustrate some numerical examples showing the achievable performance of SM-MIMO in terms of its bit error probability, signal processing complexity and energy efficiency.

In Fig. 7, the bit error probability of SM-MIMO is illustrated. The setup encompasses a MIMO transmission channel with independent and identically distributed Rayleigh fading. The receiver is equipped with $N_{r}$ receive antennas and the transmitter is configured with a single RF front-end. The target data rate is $8 \mathrm{bpcu}$. The modulation scheme and the number of transmit antennas $N_{t}$ are chosen accordingly. In addition, SM-MIMO is compared against SMX-MIMO that is configured for achieving the same data rate. The figure shows that SM-MIMO is capable 


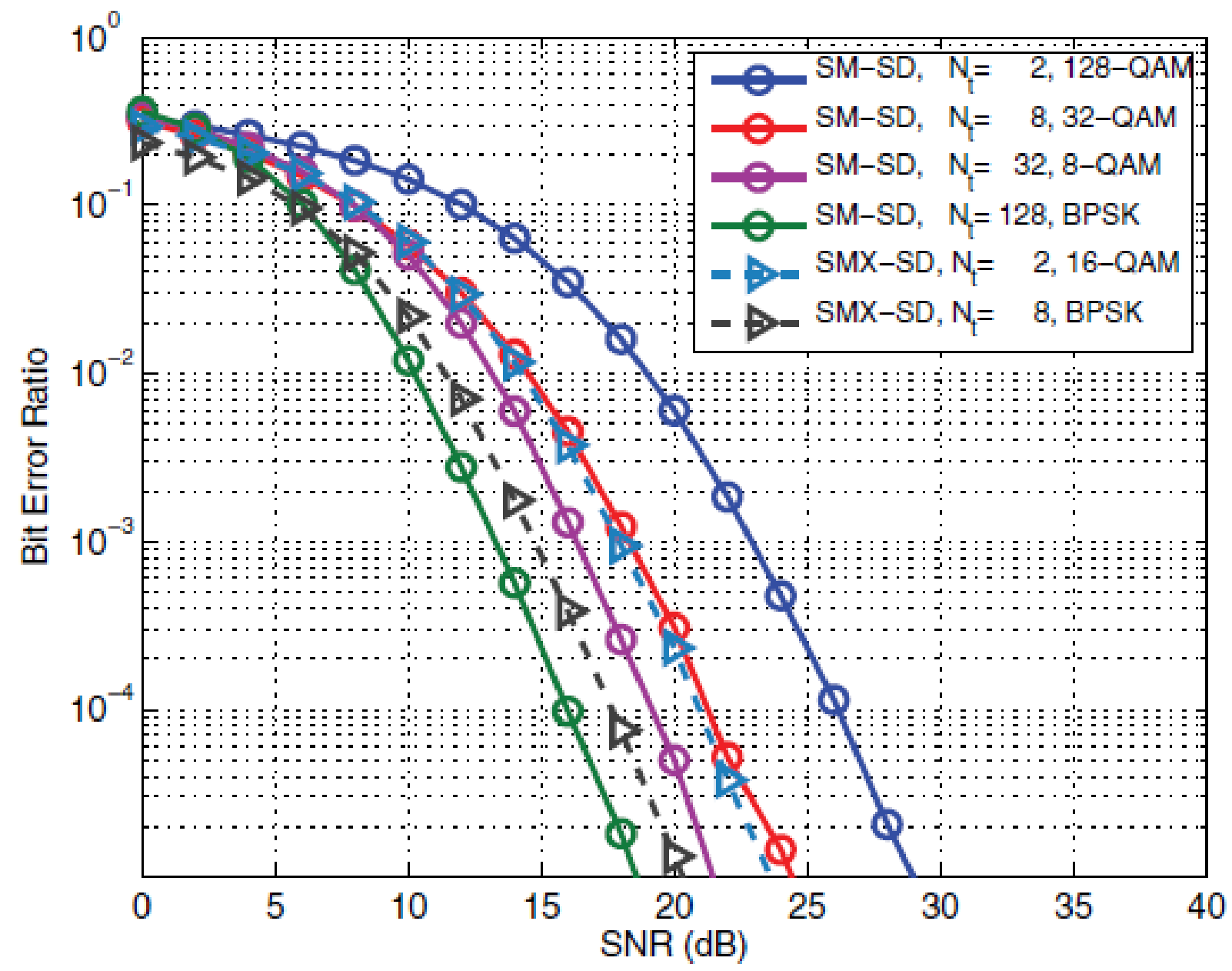

Fig. 7. Bit Error Probability. Reproduced from [40] with permission.

of providing better error performance than SMX-MIMO, under the assumption that a sufficient number of antenna-elements is available at the transmitter. Furthermore, this is achieved by using fewer RF front-ends than SMX-MIMO.

In Fig. 8, the signal processing complexity of SM-MIMO demodulation is illustrated. The same setup as in Fig. 7 is used. In particular, the demodulator is based on the Sphere Decoding (SD) principle [40] and it is shown in terms of the number of complex-valued operations with respect to the baseline ML-optimum demodulator. The figure points out that SM-MIMO is capable of offering a significant reduction of the signal processing complexity with respect to SMX-MIMO.

In Fig. 9, the EE potential of SM-MIMO is illustrated and compared against SMX-MIMO, OSTBCs (3/4 code rate) and Multiple-Input-Single-Output (SIMO) transmission for transmit- 


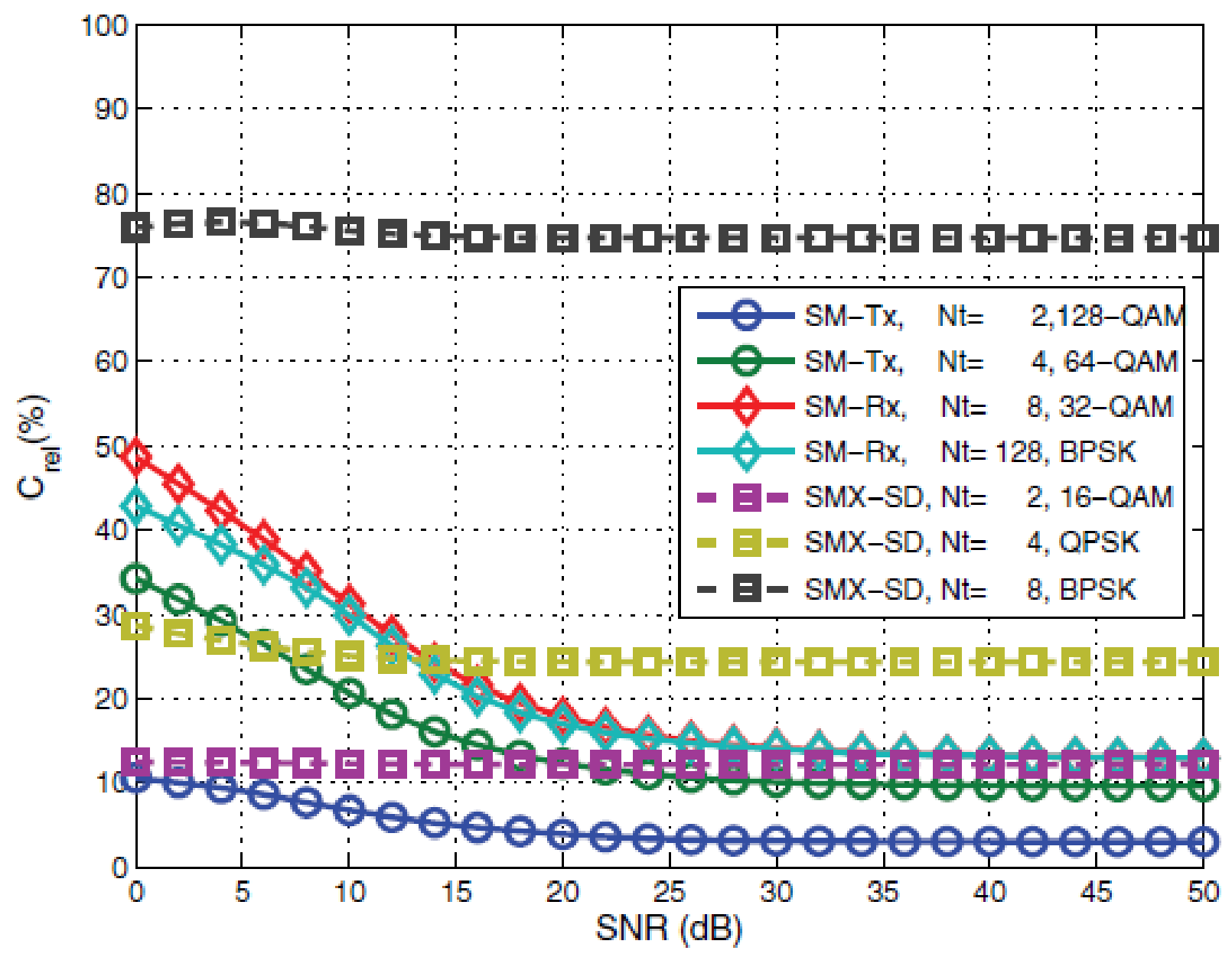

Fig. 8. Demodulation complexity. Reproduced from [40] with permission.

diversity. The EE expressed in bits/Joule is computed as the ratio between the channel capacity and the total power supply that includes both the RF and circuit power consumption. The setup encompasses a MIMO transmission channel with independent and identically distributed Rayleigh fading and four antennas at the transmitter $\left(N_{t}=4\right)$. The numerical results clearly highlight the EE potential of SM-MIMO compared to the other MIMO transmission schemes, especially for medium throughput. As for high throughput, there is an intercept point between the curves of SM-MIMO and SMX-MIMO, which is due to the limited number of TAs (only four) that is used for SM-MIMO. Increasing the number of TAs would result in further increasing the EE without the need of increasing the number of RF front-ends.

In summary, Figs. 7, 8 and 9 show that SM has the inherent potential of simultaneously 

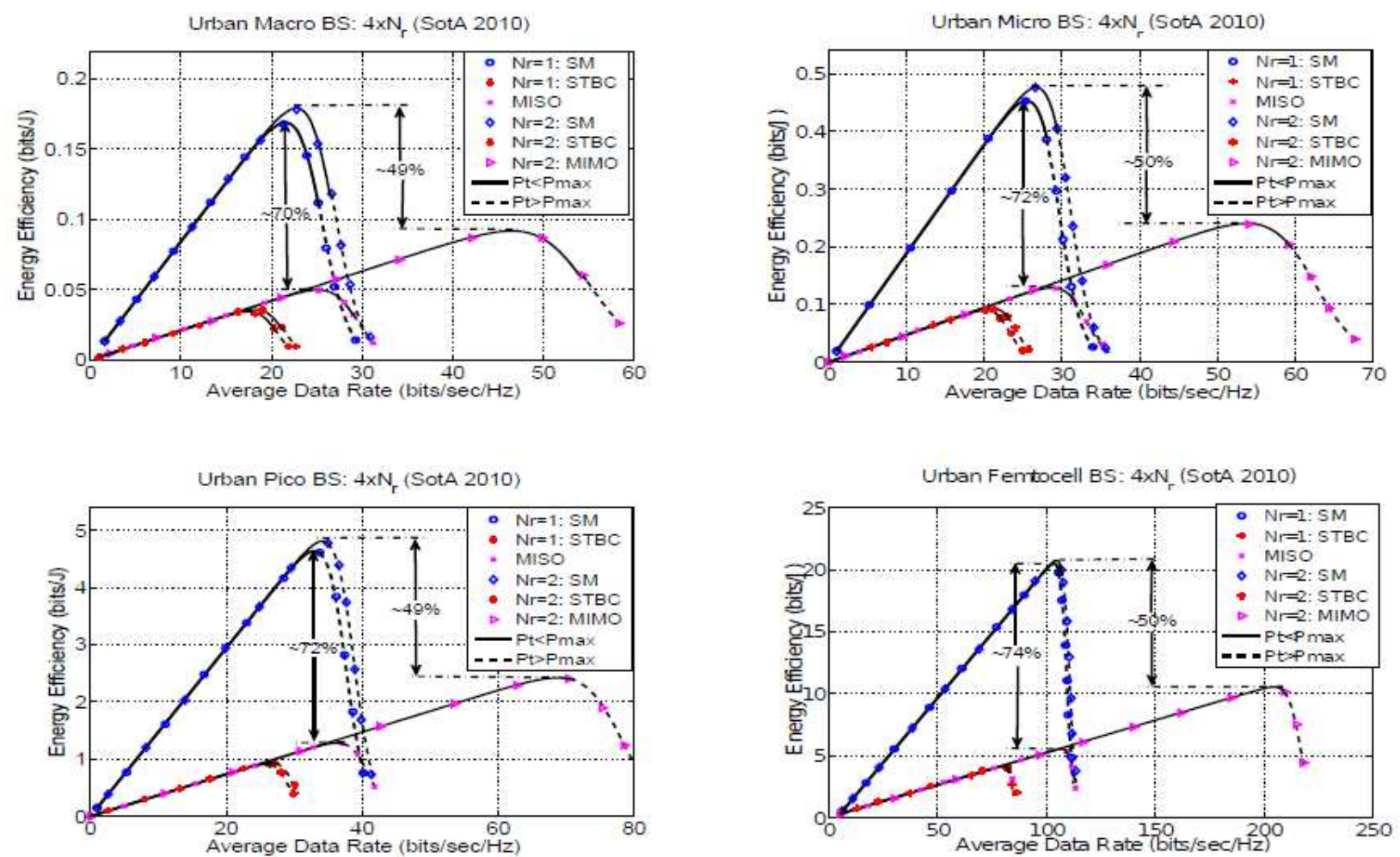

Fig. 9. Energy efficiency. Reproduced from [41] with permission.

reducing the error probability, the signal processing complexity and the power consumption of MIMO transmission. This win-win effect makes SM-MIMO a suitable candidate for emerging cellular-based NB-IoT applications [42] and realizable large-scale MIMO systems [43]. Further comparisons between SM-MIMO and state-of-the-art MIMO systems are available in [44]-[52].

\section{TESTBED IMPLEMENTATION}

In this section, we briefly describe the world's first experimental assessment of SM-MIMO transmission with the aid of a testbed operating in an indoor propagation environment.

\section{A. Transmission Chain}

The transmission chain used in the testbed setup is shown in Fig. 10, which is constituted by hardware and software parts. The hardware consists of a National Instruments (NI)-PXIe-1075 chassis at the transmitter (PXIe-Tx) and another NI-PXIe-1075 chassis at the receiver (PXIe$\mathrm{Rx}$ ), each equipped with the relevant NI modules [53]. The software carries out the digital 


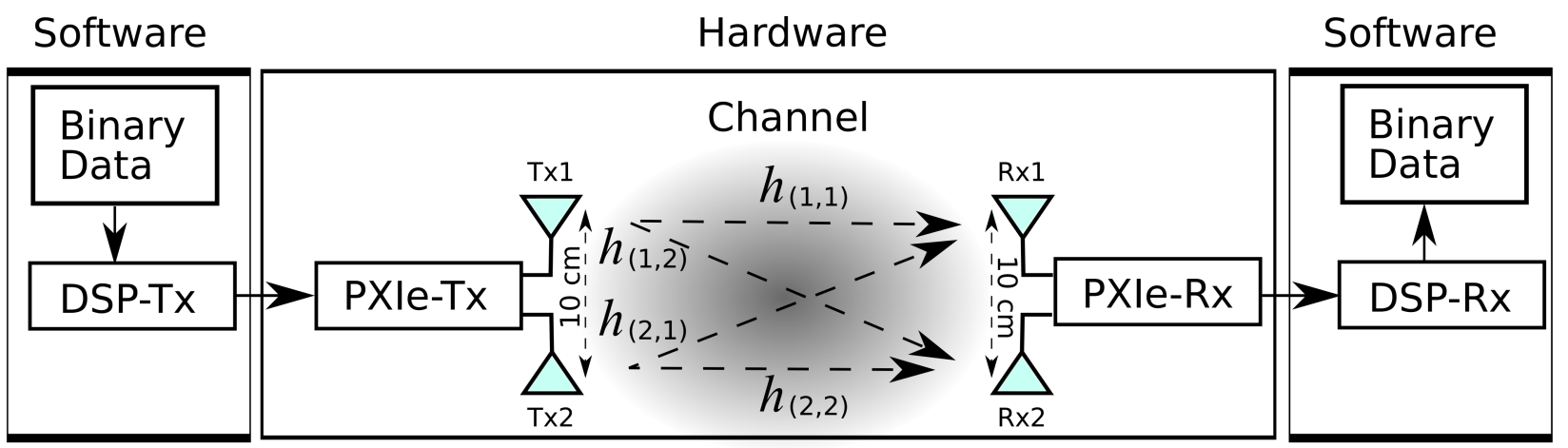

Fig. 10. Illustration of the hardware and software testbed setup. Reproduced from [54] with permission.

signal processing at the transmitter (DSP-Tx) and at the receiver (DSP-Rx). At the transmitter, the binary data is processed by the DSP-Tx block before being transmitted through the channel by the PXIe-Tx. At the receiver, the PXIe-Rx records the RF signal and outputs it to the DSP-Rx, where the original data stream is recovered.

\section{B. Hardware Testbed}

Both the NI-PXIe-1075 chassis of the transmitter and of the receiver are equipped with a 1.8 GHz Intel-i7 processor relying on 4 GB RAM. The system has two TAs and two RAs. Each TA and RA contains two quarter-wave dipoles, and a half-wave dipole placed in the middle. All three dipoles are vertically polarized. Additionally, each antenna has a peak gain of $7 \mathrm{dBi}$ in the azimuth plane, with an omnidirectional radiation pattern. The signals are transmitted at a carrier frequency of $2.3 \mathrm{GHz}$.

The PXIe-Tx transmitter hardware is composed of three interconnected hardware modules:

1) The NI-PXIe-5450 I/Q signal generator, which is fed with the transmit vector extracted from the binary file generated in MATLAB using the DSP-Tx encoding algorithm. The DSP-Tx module is described in Section V-C.

2) The NI-PXIe-5652 RF signal generator, which is connected to the NI-PXIe-5611 frequency converter, which takes its input signal from the output of the NI-PXIe-5450 I/Q signal generator.

3) The NI-PXIe-5611 frequency converter, which outputs the analog waveform corresponding to the binary data at a carrier frequency of $2.3 \mathrm{GHz}$. 


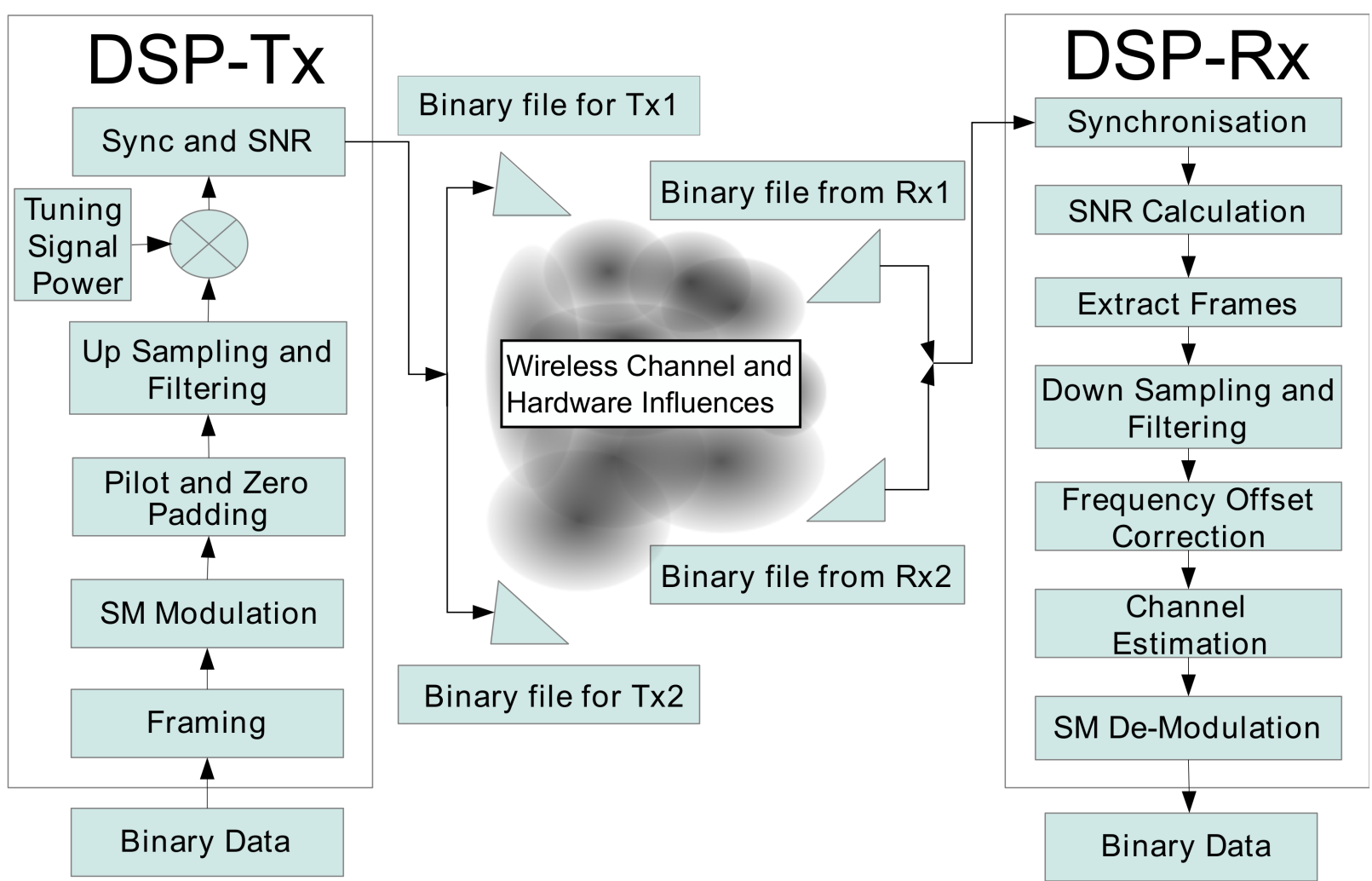

Fig. 11. Block-diagram of signal processing modules implemented at the transmitter and receiver. Reproduced from [54] with permission.

Likewise, the PXIe-Rx receiver hardware is composed of three interconnected hardware modules:

1) The NIPXIe-5601 RF down-converter, which is used for detecting the analog RF signal from the RAs.

2) The NI-PXIe-5652 on-board reference clock, which is used for synchronization.

3) The NI-PXIe-5622 IF digitizer, which applies its own bandpass filter and produces the received binary files that are processed in MATLAB by the DSP-Rx decoding algorithm. The DSP-Rx module is described in Section V-C.

\section{Software Testbed}

MATLAB is used for the digital signal processing required both at the transmitter and receiver, DSP-Tx and DSP-Rx, respectively. DSP-Tx processes the incoming information and generates a file that can be transmitted by PXIe-Tx. DSP-Rx processes the data received by PXIe-Rx and 
recovers the original binary data stream. The processing algorithms at DSP-Tx and DSP-Rx are shown in Fig. 11.

The following operations are implemented at the DSP-Tx:

1) The binary data is first split into information segments of appropriate size.

2) The information in each segment is then modulated using SM-MIMO.

3) A pilot signal is incorporated for the sake of channel estimation along with a frequency offset estimation section.

4) Then, zero-padding is performed, which permits up-sampling of the data, while maintaining the same signal power. The up-sampling ratio is set to four and the up-sampled data is passed through a root raised cosine finite impulse response filter with 40 taps and a roll-off factor of 0.75. A large roll-off factor and a long tap-delay are necessary for ensuring that the power is focused to a short time window, i.e., for ensuring that only a single antenna is activated in every channel use.

5) The resultant vector is multiplied by a factor termed as the "Tuning Signal Power" for the sake of adjusting the desired transmit power required for the information sequence.

6) The frames are created by ensuring that the frame length multiplied by the sampling rate is less than the coherence time of the channel, which is typically about $7 \mathrm{~ms}$ for a stationary indoor environment. This ensures that all channel estimates generated at the receiver are valid for the frame duration.

The following operations are implemented at DSP-Rx:

1) The raw data vectors received from the NI-PXIe-5622 digitizer are combined, in order to form the received matrix for data demodulation.

2) The detector first finds the beginning of the transmitted sequence by using the synchronization sequence (based on an autocorrelation algorithm).

3) The Signal-to-Noise-Ratio (SNR) for each vector is calculated using the "SNR section".

4) Each raw vector is decomposed into its underlying frames.

5) Each frame is down-sampled and passed through a root raised cosine filter which completes the matched-filtering.

6) Frequency offset estimation, timing recovery and correction of each frame follow, which rely on using state-of-the-art algorithms. 


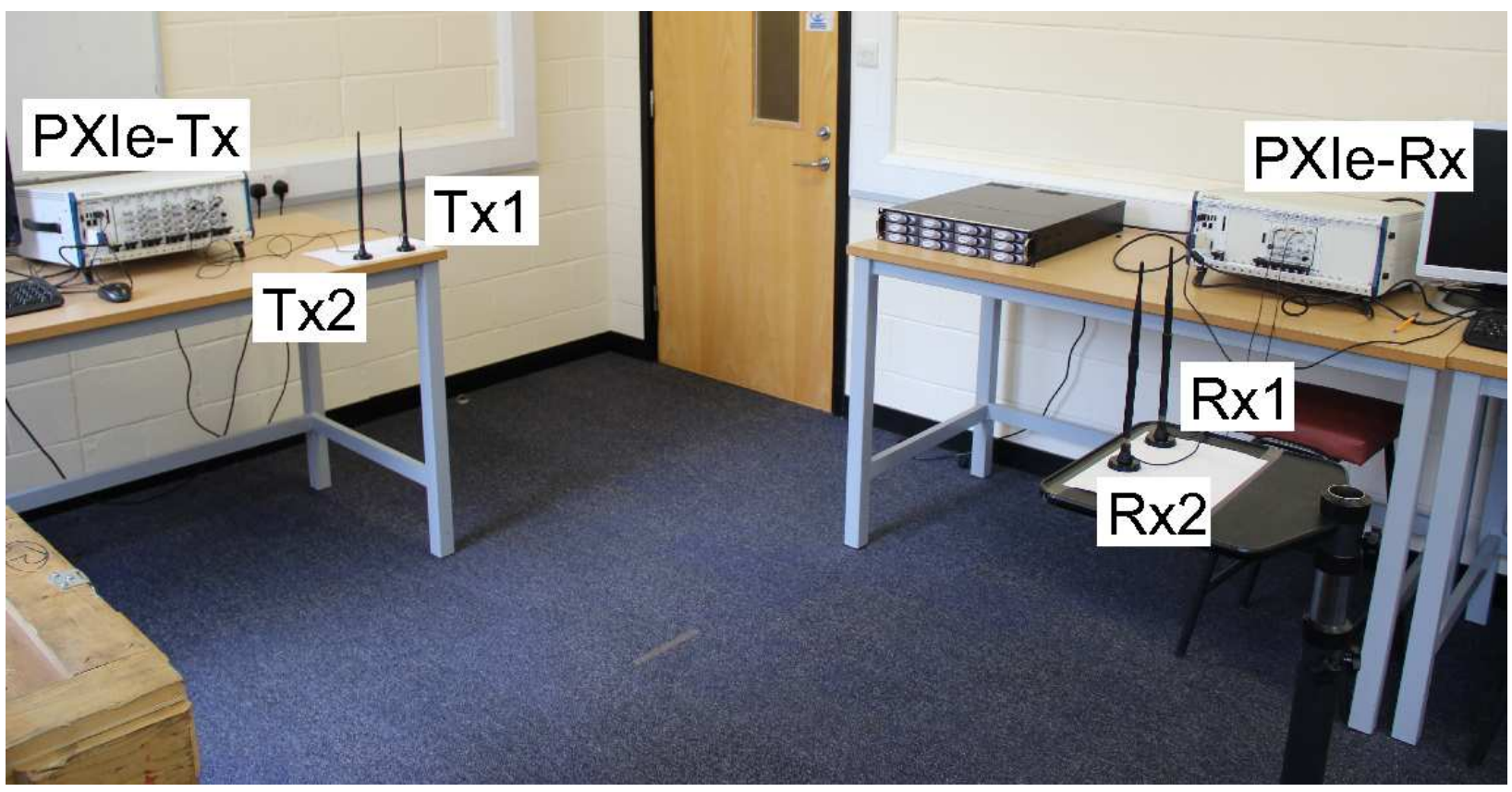

Fig. 12. Illustration of the laboratory setup. Reproduced from [54] with permission.

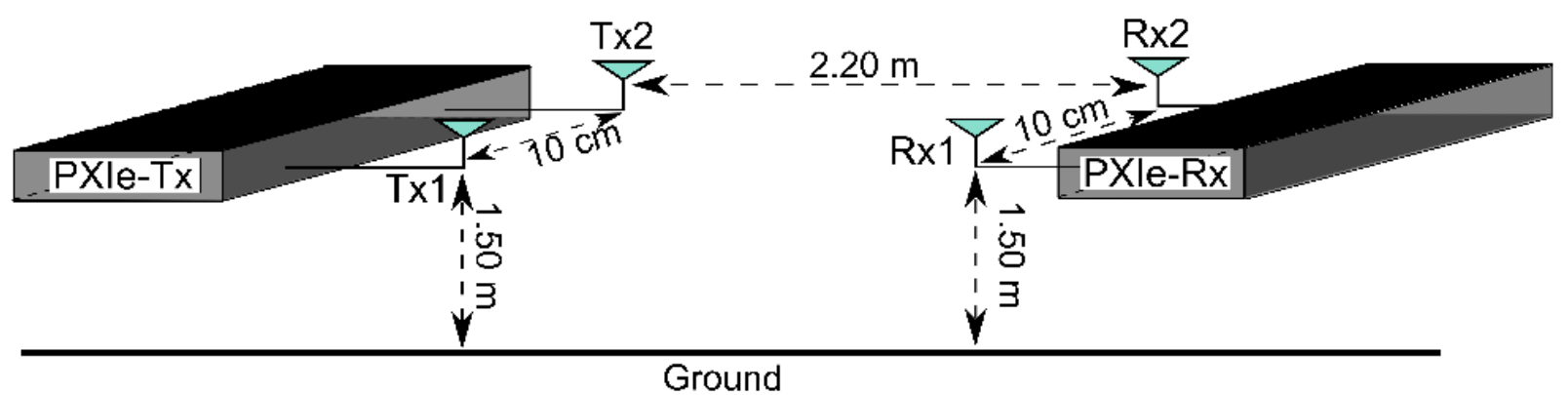

Fig. 13. Illustration of the measurement setup. The pair of receive and transmit antennas are set $2.2 \mathrm{~m}$ apart from each other with a direct line-of-sight. Each pair of antennas is $1.5 \mathrm{~m}$ above the ground floor. The spacing between the antennas in either pair is $10 \mathrm{~cm}$, which corresponds to 0.77 times the wavelength at $2.3 \mathrm{GHz}$. Reproduced from [54] with permission.

7) The pilot signal is then used for channel estimation.

8) The remaining data, along with the estimated channels, is finally used for recovering the estimated binary sequence (ML-optimum demodulation).

\section{Experimental Evaluation}

The physical layout of the experimental setup is shown in Fig. 12, and the relative antenna spacing is provided in Fig. 13. More particularly, the two TAs and two RAs are identical and are 


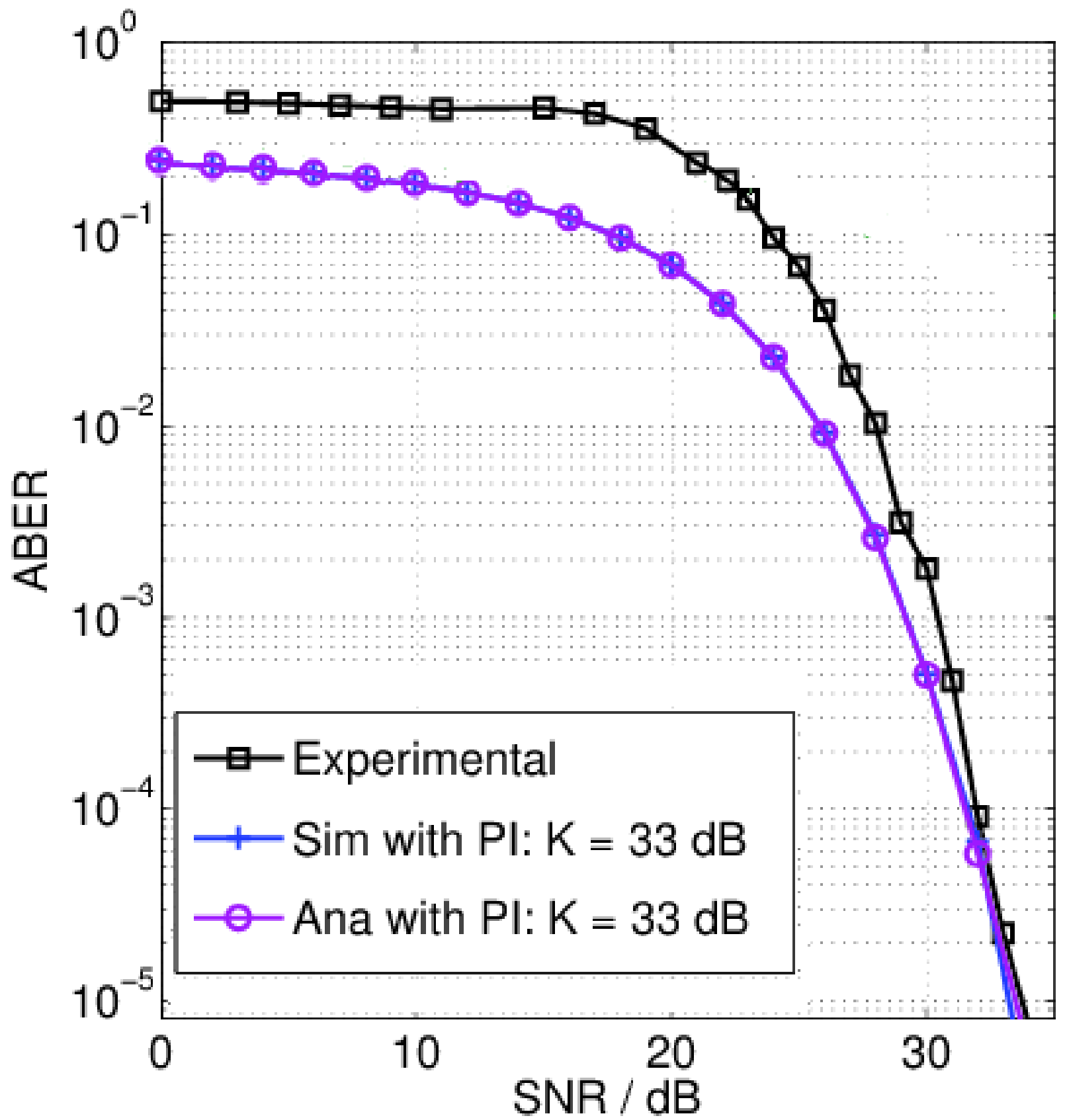

Fig. 14. Illustration of the experimental results and comparison with analysis and simulations. Reproduced from [54] with permission.

placed directly across each other. As such, the channel between the transmitter and receiver has a strong LoS component. Therefore, the transmit-to-receive channel exhibits a Rician distribution with a high Rician factor due to the short distance between transmitter and receiver. In order to confirm the Rician distribution and to estimate the Rician factor, some channel measurements are 
collected and studied with the help of the Chi-squared goodness of fit test. These measurements confirm that the channel is Rician distributed with a Rician factor in the range of 31-38 dB.

To assess the performance of the testbed, some measurements are performed and the error probability is computed experimentally. Specifically, a stream of $10^{4}$ information bits is transmitted. This data is split into 50 frames with 200 bits each. To implement coherent detection, the channel is estimated both at the beginning and at the end of every frame, resulting in 100 CEs per transmission. The experiment is repeated 1000 times for every SNR point and the error probability is computed. The results are shown in Fig. 14. A good agreement between the experiments and both the Monte Carlo simulations ("Sim") and the analytical results ("Ana") can be observed. Explicitly, the analytical results are obtained by considering a Rician distribution with a Rician factor equal to $33 \mathrm{~dB}$ and by taking into account some of the associated hardware imperfections, such as the power imbalance of the RF front-ends. The gap observed at low SNR can be attributed to a number of practical factors, including the imperfect frequency offset estimation, timing recovery errors, synchronization problems and inaccurate channel estimation.

In summary, the preliminary results illustrated in this section for a simple $(2 \times 2)$-element MIMO testbed, including full transmit and reception chains and signal processing algorithms, confirm that the theoretical gains predicted by the analysis and simulations are substantiated by measurements performed in a controlled laboratory environment. Further experimental activities on SM-MIMO are available in [55].

\section{Conclusions and Research Outlook}

The emerging market of the IoT requires novel power-efficient and low-complexity MIMOaided cellular radio access technologies. This trend will have a profound impact on both the theory and practice of future cellular networks, which will no longer be purely optimized for approaching the attainable capacity, but will explicitly include the energy efficiency during the design and optimization of the entire protocol stack. In this chapter, we have critically appraised SM, which constitutes a promising transmission concept in the context of MIMO-aided transmission. We have shown that the SM concept can be beneficially invoked for the design of medium rate, low-complexity and energy-efficient communication systems by relying on a limited number of RF chains. Preliminary experimental results substantiating the benefits of SMMIMO have also been illustrated. Promising research activities on SM-MIMMO are constituted 
by amalgamating the SM concept with reconfigurable antennas, aiming for the design of cellular systems supporting low-cost, low-complexity, low-power consumption and medium-throughput IoT applications [42].

We close this chapter by directing interested readers to a collection of companion tutorial slides [56] and a tutorial YouTube video [57] on SM-MIMO research.

\section{REFERENCES}

[1] http://www.3gpp.org/lte-advanced.

[2] A. Ghosh, J. Zhang, J. G. Andrews, and R. Muhamed, Fundamentals of LTE, Prentice-Hall, June 2010.

[3] Z. Hasan, H. Boostanimehr, and V. K. Bhargava, "Green cellular networks: A survey, some research issues and challenges," IEEE Commun. Surveys Tuts., vol. 13, no. 4, pp. 524-540, Nov. 2011.

[4] M. Di Renzo, L. Alonso, F. H. P. Fitzek, A. Foglar, F. Granelli, F. Graziolsky, C. Gruet, H. Haas, G. Kormentzas, A. I. Perez, J. Rodriguez, J. Thompson, and C. Verikoukis, "GREENET - An early stage training network in enabling technologies for green radio," IEEE Veh. Technol. Conf. - Workshops, pp. 1-5, May 2011.

[5] 3GPP TSG-SA\#50 SP-1008883GPP, 3GPP Work Item Description, Study on system enhancements for energy efficiency, 3GPP TSGSA, Istanbul, Turkey, Tech. Rep., Dec. 2010, agreed Work Item 500037 (Release 11).

[6] 3GPP Narrow-Band Internet of Things. [Online]. Available: http://www.3gpp.org/news-events/3gpp-news/1733-niot.

[7] J. Mietzner, R. Schober, L. Lampe, W. H. Gerstacker, and P. A. Hoeher, "Multiple-antenna techniques for wireless communications - A comprehensive literature survey," IEEE Commun. Surveys Tuts., vol. 11, no. 2, pp. 87-105, 2nd quarter 2009.

[8] H. Huang, C. B. Papadias, and S. Venkatesan, MIMO Communication for Cellular Networks, Springer, Nov. 2011.

[9] S. D. Gray, "Theoretical and practical considerations for the design of green radio networks," IEEE Veh. Technol. Conf. Spring, Keynote speech, May 2011. [Online]. Available: http://www.ieeevtc.org/conf-admin/vtc2011spring/5.pdf.

[10] G. Auer, V. Giannini, I. Godor, P. Skillermark, M. Olsson, M. Imran, D. Sabella, M. Gonzalez, C. Desset, and O. Blume, “Cellular energy efficiency evaluation framework," IEEE Veh. Technol. Conf. - Spring, pp. 1-6, May 2011.

[11] G. Auer, O. Blume, V. Giannini, I. Godor, M. Imran, Y. Jading, E. Katranaras, M. Olsson, D. Sabella, P. Skillermark, and W. Wajda, "D2.3: Energy efficiency analysis of the reference systems, areas of improvements and target breakdown," EARTH: Energy Aware Radio and neTwork tecHnologies, Jan. 2012. [Online]. Available: https://bscw.ictearth.eu/pub/bscw.cgi/d71252/EARTH_WP2_D2.3_v2.pdf.

[12] F. Heliot, M. A. Imran, and R. Tafazolli, "On the energy efficiency-spectral efficiency trade-off over the MIMO Rayleigh fading channel," IEEE Trans. Commun., vol. 60, no. 5, pp. 1345-1356, May 2012.

[13] A. Mohammadi and F. M. Ghannouchi, "Single RF front-end MIMO transceivers," IEEE Commun. Mag., vol. 49, no. 12, pp. 104-109, Dec. 2011.

[14] M. Di Renzo, H. Haas, and P. M. Grant, "Spatial modulation for multiple-antenna wireless systems: A survey," IEEE Commun. Mag., vol. 49, no. 12, pp. 182-191, Dec. 2011.

[15] Y. Chau and S.-H. Yu, "Space modulation on wireless fading channels," IEEE Veh. Technol. Conf. - Fall, pp. 1668-1671, Oct. 2001.

[16] H. Haas, E. Costa, and E. Schultz, "Increasing spectral efficiency by data multiplexing using antennas arrays," IEEE Int. Symp. Personal, Indoor, Mobile Radio Commun., pp. 610-613, Sep. 2002. 
[17] S. Song, Y. L. Yang, Q. Xiong, K. Xie, B.-J. Jeong, and B. L. Jiao, "A channel hopping technique I: Theoretical studies on band efficiency and capacity," IEEE Int. Conf. Commun., Circuits and Systems, pp. 229-233, June 2004.

[18] R. Y. Mesleh, H. Haas, C. W. Ahn, and S. Yun "Interchannel interference avoidance in MIMO transmission by exploiting spatial information,” IEEE Int. Symp. Personal, Indoor, Mobile Radio Commun., pp. 141-145, Sep. 2005.

[19] R. Y. Mesleh, H. Haas, C. W. Ahn, and S. Yun, "Spatial modulation - OFDM," Int. OFDM Workshop, pp. 288-292, Aug. 2006.

[20] S. Ganesan, R. Y. Mesleh, H. Haas, C. W. Ahn, and S. Yun, "On the performance of spatial modulation OFDM," Asilomar Conf. Signals, Systems Computers, pp. 1825-1829, Oct. 2006.

[21] R. Y. Mesleh, H. Haas, C. W. Ahn, and S. Yun, "Spatial modulation - A new low complexity spectral efficiency enhancing technique," IEEE Int. Conf. Commun. Netw. in China, pp. 1-5, Oct. 2006.

[22] R. Y. Mesleh, S. Ganesan, and H. Haas, "Impact of channel imperfections on spatial modulation OFDM," IEEE Int. Conf. Commun. Netw. in China, pp. 1-5, Sep. 2007.

[23] Y. Yang and B. Jiao, "Information-guided channel-hopping for high data rate wireless communication," IEEE Commun. Lett., vol. 12, no. 4, pp. 225-227, Apr. 2008.

[24] R. Y. Mesleh, H. Haas, S. Sinanovic, C. W. Ahn, and S. Yun, "Spatial modulation," IEEE Trans. Veh. Technol., vol. 57, no. 4, pp. 2228-2241, July 2008.

[25] J. Jeganathan, A. Ghrayeb, and L. Szczecinski, "Spatial modulation: Optimal detection and performance analysis," IEEE Commun. Lett., vol. 12, no. 8, pp. 545-547, Aug. 2008.

[26] J. Jeganathan, A. Ghrayeb, L. Szczecinski, and A. Ceron, "Space shift keying modulation for MIMO channels," IEEE Trans. Wireless Commun., vol. 8, no. 7, pp. 3692-3703, July 2009.

[27] R. Y. Mesleh, S. Engelken, S. Sinanovic, and H. Haas, “Analytical SER calculation of spatial modulation,” IEEE Int. Symp. Spread Spectrum Tech. Applic., pp. 272-276, Aug. 2008.

[28] J. Jeganathan, A. Ghrayeb, and L. Szczecinski, "Generalized space shift keying modulation for MIMO channels," IEEE Int. Symp. Personal, Indoor, Mobile Radio Commun., pp. 1-5, Sep. 2008.

[29] S. Sugiura, "Coherent versus non-coherent reconfigurable antenna aided virtual MIMO systems," IEEE Signal Processing Letters, vol. 21, no. 4, pp. 390-394, April 2014.

[30] Z. Bouida, H. El-Sallabi, A. Ghrayeb, and K. A. Qaraqe, "Enhanced Space-Shift Keying (SSK) with Reconfigurable Antennas,” IEEE Int. Conf. on Commun. (ICC'15), London, U.K, June 2015.

[31] Z. Bouida, H. El-Sallabi, A. Ghrayeb, and K. A. Qaraqe, "Reconfigurable Antenna-based Space-Shift Keying (SSK) for MIMO Channels," IEEE Trans. Commun., vol. 15, no. 1, pp. 446-457, January 2016.

[32] Z. Bouida, H. El-Sallabi, M. Abdallah, A. Ghrayeb, and K. A. Qaraqe, "Reconfigurable Antenna-Based Space-Shift Keying for Spectrum Sharing Systems," IEEE Int. Conf. on Commun. (ICC'16), Kuala Lumpur, Malaysia, May 2016.

[33] Z. Bouida, H. El-Sallabi, M. Abdallah, A. Ghrayeb, and K. A. Qaraqe, "Reconfigurable Antenna-Based Space-Shift Keying for Spectrum Sharing Systems under Rician Fading," IEEE Trans. Wireless Commun., $2^{\text {nd }}$ round of reviews, February 2016.

[34] M. Di Renzo, H. Haas, A. Ghrayeb, S. Sugiura, and L. Hanzo, "Spatial modulation for generalized MIMO: Challenges, opportunities and implementation," Proc. of the IEEE, vol. 102, no. 1, pp. 56-103, Jan. 2014.

[35] S. Sugiura, S. Chen, and L. Hanzo, "A universal space-time architecture for multiple-antenna aided systems," IEEE Commun. Surveys Tuts., vol. 14, no. 2, pp. 401-420, 2nd quarter 2012.

[36] P. Yang, M. Di Renzo, Y. Xiao, S. Li, and L. Hanzo, "Design guidelines for spatial modulation," IEEE Commun. Surveys Tuts., vol. 16, no. 1, pp. 6-26, 1st quarter 2015. 
[37] P. Yang, Y. Xiao, Y. L. Guan, K. V. S. Hari, A. Chockalingam, S. Sugiura, H. Haas, M. Di Renzo, C. Masouros, Z. Liu, L. Xiao, S. Li, and L. Hanzo, "Single-carrier SM-MIMO: A promising design for broadband large-scale antenna systems," IEEE Commun. Surveys Tuts., to appear.

[38] S. M. Alamouti, "A simple transmit diversity technique for wireless communications," IEEE J. Sel. Areas Commun., vol. 16, no. 8, pp. 1451-1458, Oct. 1998.

[39] V. Tarokh, H. Jafarkhani, and R. Calderbank, "Space-time block codes from orthogonal designs," IEEE Trans. Inform. Theory, vol. 45, no. 5, pp. 1456-1467, July 1999.

[40] A. Younis, S. Sinanovic, M. Di Renzo, R. Y. Mesleh, and H. Haas, "Generalised sphere decoding for spatial modulation," IEEE Trans. Commun., vol. 61, no. 7, pp. 2805-2815, July 2013.

[41] A. Stavridis, S. Sinanovic, M. Di Renzo, and H. Haas, "Energy evaluation of spatial modulation at a multi-antenna base station,” IEEE Veh. Technol. Conf. - Fall, pp. 1-5, Sep. 2013.

[42] ANR-2015, "Modulation spatiale et antenne reconfigurable pour connecter en haut debit des capteurs mobiles et autonomes en energie," [Online]. Available: http://www.agence-nationale-recherche.fr/fileadmin/aap/2015/selection/pa-anr2015-selection-DS07.pdf.

[43] 5G-PPP, "White paper for research beyond 5G," [Online]. Available: http://networld2020.eu/wpcontent/uploads/2015/11/B5G-Vision-for-Researchv-1.0-for-public-consultation.pdf.

[44] M. Di Renzo and H. Haas, "Space shift keying (SSK) modulation: On the transmit-diversity/multiplexing trade-off," IEEE Int. Conf. Commun., pp. 1-6, June 2011.

[45] M. Di Renzo and H. Haas, "Transmit-diversity for spatial modulation (SM): Towards the design of high-rate spatiallymodulated space-time block codes," IEEE Int. Conf. Commun., pp. 1-6, June 2011.

[46] M. Di Renzo and H. Haas, "Bit error probability of space modulation over Nakagami-m fading: Asymptotic analysis," IEEE Commun. Lett., vol. 15, no. 10, pp. 1026-1028, Oct. 2011.

[47] M. Di Renzo and H. Haas, "Bit error probability of SM-MIMO over generalized fading channels," IEEE Trans. Veh. Technol., vol. 61, no. 3, pp. 1124-1144, Mar. 2012.

[48] N. Serafimovski, S. Sinanovic, M. Di Renzo, and H. Haas, "Multiple access spatial modulation,” EURASIP J. Wireless Commun. and Networking, Sep. 2012.

[49] M. Di Renzo and H. Haas, "On transmit-diversity for spatial modulation MIMO: Impact of spatial-constellation diagram and shaping filters at the transmitter," IEEE Trans. Veh. Technol., vol. 62, no. 6, pp. 2507-2531, July 2013.

[50] M.-T. Le, V.-D. Ngo, H.-A. Mai, X.-N. Tran, and M. Di Renzo, "Spatially modulated orthogonal space-time block codes with nonvanishing determinants," IEEE Trans. Commun., vol. 62, no. 1, pp. 85-99, Jan. 2014.

[51] W. Lu and M. Di Renzo "Performance analysis of spatial modulation MIMO in a Poisson field of interferers," IEEE Int. Conf. Comput., Netw., Commun., pp. 1-7, Feb. 2014.

[52] A. Stavridis, S. Sinanovic, M. Di Renzo, H. Haas, and P. M. Grant, "An energy saving base station employing spatial modulation," IEEE Int. Workshop on Computer-Aided Modeling Analysis and Design of Communication Links and Networks, pp. 1-6, Sep. 2012.

[53] National Instruments, "NI PXIe-1075 User Manual and Specifications". [Online]. Available: http://www.ni.com/pdf/manuals/372537b.pdf.

[54] N. Serafimovski, A. Younis, R. Mesleh, P. Chambers, M. Di Renzo, C.-X. Wang, P. M. Grant, M. A. Beach, and H. Haas, "Practical implementation of spatial modulation," IEEE Trans. Veh. Technol., vol. 62, no. 9, pp. 4511-4523, Sep. 2013. 
[55] A. Younis, W. Thompson, M. Di Renzo, C.-X. Wang, M. A. Beach, H. Haas, and P. M. Grant, "Performance of spatial modulation using measured real-world channels," IEEE Veh. Technol. Conf. - Fall, pp. 1-5, Sep. 2013.

[56] Spatial modulation for generalized MIMO: Challenges, opportunities and implementation. [Online]. Available: http://halsupelec.archives-ouvertes.fr/docs/00/87/50/26/ANNEX/ProcIEEE_SMPaper_Slides.pdf.

[57] Spatial modulation for generalized MIMO: Challenges, opportunities and implementation. [Online]. Available: http://www.youtube.com/watch?v=cNgCJK4oimM\&feature=youtu.be. 